\title{
Child and Adult Autism Spectrum Disorder in COVID-19 Pandemic
}

Marcos Altable

Funding: The author(s) received no specific funding for this work.

Potential competing interests: The author(s) declared that no potential competing interests exist.

\begin{abstract}
The worldwide population prevalence of autism is about $1 \%$. The current panorama that we are experiencing with the COVID-19 pandemic, makes it difficult for many people to understand and assimilate everything what is happening. In persons with autism, this difficulty can become extreme, both in children and adults. However, there are some recommendations to help process and assimilate this situation and like this avoid or reduce complications in this population. This recommendations are simple, but they are difficult to perform. However, they can help keep the loved ones and their family members more bearable. Only by knowing its characteristics and applying a few recommendations or guidelines, caregivers and the affected persons will be able to lead a more enjoyable life.
\end{abstract}

The worldwide population prevalence of autism is about 1\% (Lai et al., 2019). The current panorama that we are experiencing with the COVID-19 pandemic, makes it difficult for many people to understand and assimilate everything what is happening. In persons with autism, this difficulty can become extreme, both in children and adults. Approximately $10 \%$ of autistic people have co-occurring physical disabilities (Rydzewska et al., 2019). An increased rates of various chronic illnesses and medical conditions have been observed in persons whit autism (Neumeyer et al., 2019). And also, atypical immune responses are showed (Meltzer \& Van De Water, 2017). All these factors may increase the vulnerability to COVID-19. Recent evidence suggests that experiencing to a pandemic carries symptoms of anxiety, depression, and posttraumatic stress disorder (Jalloh et al., 2018). Since autistic people are overwhelmingly likely to experience mental illness (Cvejic, Arnold, Foley, \& Trollor, 2018; Lever \& Geurts, 2016) and nine times more likely than the general population to die by suicide (Hirvikoski et al., 2016), the mental health consequences of COVID-19 may be devastating (den Houting, 2020).

Autism spectrum, also known as autism spectrum disorder (ASD), is a range of mental 
disorders of the neurodevelopmental type in the areas of the social communication, restricted interests, and repetitive behaviours (American Psychiatric Association, 2013). Individuals with autism have atypical cognitive profiles, such as impaired social cog nition and social perception, executive dysfunction, and atypical perceptual and information processing (Lai et al., 2019). The practice of isolation and quarantine, an important preventive measure, carries implications in civil liberty and clinical practice (Drogomyretska, Fox, \& Colbert, 2020). Given the clear difficulties arising from the pandemic that present for people with ASD, they and their caregivers may be seriously affected (Drogomyretska et al., 2020). All aspects of confinement may influence on living complications in different ways. However, there are some recommendations to help process and assimilate this situation and like this avoid or reduce complications in this population.

Routine and firmness are essential for people with autism. Even the smallest and most common changes can be annoying. COVID-19 has brought the end of routine, school activity, special programs at school, day programs and, in some cases, support at work. Most persons who suffer for autism participate in multiple therapy ways (speech, occupational, physical, social, behavioral, and/or psychological). In most cases, continuing with these therapies is impossible.

Difficulties in sensory perception is one of the most important aspects to consider in eating problems in people with autism. Many of them may have taste and tactile hypersensitivity or hypersensitivity. The touch system is responsible for making us feel the temperature and notice the different textures of the food. In childhood, observing the different responses of the child may indicate possible problems related to taste and touch sensory processing and will help us to respond to these situations. Therefore, many autistic people are unusually picky about the foods they eat. Their diet may consist of specific foods or only certain brand names. During the currently crisis, it is difficult to go to a grocery store, let alone find a specific brand of a specific food. Lack of familiar foods, known to their daily routine, can be incredibly stressful in the ASD.

All of them trust their relationships with specific teachers, therapists, family members and caregivers (Roorda, Koomen, Spilt, Thijs, \& Oort, 2013). During this health crisis, access to such persons may be limited or non-existent, which can contribute to an increase in stress and behavior problems.

Some people on the autism spectrum live in settings outside the home and, just like in nursing homes, these settings are now considered "prohibited" for family members. This lack of interaction with acquaintances can be stressful for everyone. The COVID-19 pandemic poses challenges for families with members who suffer from autism. When these family members struggle with stress and anxiety, people around 
them are likely to do so as well (Lai et al., 2019). They struggle to maintain routine, since all people with autism need routine and consistency. This is difficult (or impossible) to provide if you are trapped at home, working from home, supporting other children's education, or dealing with an illness (Narzisi, 2020).

There are problems in communicating the current situation. It can be difficult to explain the COVID-19 pandemic to some persons with autism who may have difficulties with receptive speech or may have intellectual disabilities.

On the other hand, while all children and parents are strugg ling with education at this time, it is particularly difficult for families with special needs to access the programs, educational supports and other resources to which they are entitled.

How to support your loved one with autism?

It may take a long time for a loved one with autism to return to their daily activities and routines. To live and be able to carry this, parents and other family members must establish and maintain a lifestyle at home that works well enough for everyone.

\section{Help understand what is happening}

Not all children or adults with autism can understand the details of a global viral pandemic, but the vast majority can understand the basics.

Teach safety tips

Make sure that the person with autism learns how to maintain proper hygiene. This is through frequent handwashing, lasting at least 20 seconds, covering your nose and mouth when coughing or sneezing, maintaining social distance and wearing a mask when in public. If these measures were difficult for them to apply, consider staying away from stores and other group settings during this time.

Maintain social connections

Many people with autism are used to interacting regularly with family, friends, or support staff, so communication via the internet or by phone can be used to maintain these important relationships (Kumazaki et al., 2020).

\section{Establish a routine}

Most people with autism or not, work best with a set routine and schedule, but it can be difficult to stick to if it is not necessary for work or school. For individuals with autism, a routine can make the difference between a calm and pleasant family life and days full of emotional crisis and outbursts. Routines should not be complex, they just must be consistent. Establish regular mealtimes and help the family member with autism notice when it is time to prepare to eat. The use of timers and alarms can be useful, so that the autistic family member knows what to expect and when to expect it. It is also important to try to offer familiar and preferred foods in this stressful moment. It is recommended to assign a working time. If the family member whit autism is of 
school age, it is a good idea to set aside relatively short periods of time (30-60 minutes) to focus on schoolwork. If he is an adult, consider assigning specific tasks or projects that interest him, such as cleaning, cooking, folding and doing laundry, putting away dishes, etc. during these time periods.

Another important point is to maintain a rigidity at bedtime and wake up, since lack of sleep (or excess sleep) can be a big problem for a person with autism. If exist sleep problems, consider using a melatonin supplement.

Reserve time for fun, choosing certain times of the day for activities as television, games and social networks. Setting alarms to mark the beginning and end of those moments, so that they become part of the daily routine.

Provide relaxing resources

Many persons with autism have extremely sensitive sensory systems and may need a wide repertoire of resources to stay calm (Kojovic, Ben Hadid, Franchini, \& Schaer, 2019). They may also need help to maintain their emotions. Some options include procure quiet space, create or take advantage of opportunities for stimulation, and physical exercise. Consider take a walk with family, playing or walking in the backyard, climbing stairs, dancing, etc. (Boucenna et al., 2014; Peckett, Maccallum, \& Knibbs, 2016) Sensory resources for people with autism often include to receive sensory regimens, guidelines or activities from an occupational therapist. The therapist should be asked how to maintain sensory activities using balls, toys, swings, blankets or other means that may be at home.

\section{Drugs}

If they are taking any drugs, make sure you are taking your medicine regularly. Anyone with a school-age child with autism has the right to take advantage of special education resources throughout their city. If this support is not being received or is not appropriate, feel free to contact your child's teacher, principal, or therapist.

Caregivers of a person with autism during COVID-19 faces an unusually challenging situation. It would be advisable to take measures to ensure your own well-being (a walk to disconnect and recharge, meditation in the early morning, ask for help if necessary or there is an overload), it is mean to do everything possible to take it easy. Furthermore, this will be transmitted to the person with autism.

It should be noted that in adults, it is common a greater social integration, since they have learned certain compensatory social skills. Specially in mild or moderate cases. Economic independence and the completion of professional or university studies, it is something that children do not consider. Adolescence is particularly problematic, as it is a difficult stage in itself.

At the present time of isolation and uncertainty, both caregivers and educational staff 
and, the individuals with ASD face difficult challenges. Autism is already an exceedingly difficult to handle disorder, and given the peculiarities of this population, they are extremely vulnerable during a pandemic. Only by knowing its characteristics and applying a few recommendations or guidelines, caregivers and the affected persons will be able to lead a more enjoyable life.

\section{REFERENCES}

American Psychiatric Association. (2013). DSM-5 Diagnostic Classification. In Diagnostic and Statistical Manual of Mental Disorders. https://doi.org/10.1176/appi.books.9780890425596.x00diagnosticclassification Boucenna, S., Narzisi, A., T ilmont, E., Muratori, F., Pioggia, G., Cohen, D., \& Chetouani, M. (2014). Interactive Technologies for Autistic Children: A Review. Cognitive Computation, 6(4), 722-740. https://doi.org/10.1007/s12559-014-9276-x

Cvejic, R. C., Arnold, S. R. C., Foley, K.-R., \& Trollor, J. N. (2018). Neuropsychiatric profile and psychotropic medication use in adults with autism spectrum disorder: results from the Australian Long itudinal Study of Adults with Autism. BJPsych Open, 4(6), 461-466. https://doi.org/10.1192/bjo.2018.64 den Houting, J. (2020). Stepping Out of Isolation: Autistic People and COVID-19. Autism in Adulthood, aut.2020.29012.jdh. https://doi.org/10.1089/aut.2020.29012.jdh

Drogomyretska, K., Fox, R., \& Colbert, D. (2020). Brief Report: Stress and Perceived Social Support in Parents of Children with ASD. Journal of Autism and Developmental Disorders. https://doi.org/10.1007/s10803-020-04455-x Hirvikoski, T., Mittendorfer-Rutz, E., Boman, M., Larsson, H., Lichtenstein, P., \& Bölte, S. (2016). Premature mortality in autism spectrum disorder. British Journal of Psychiatry, 208(3), 232-238. https://doi.org/10.1192/bjp.bp.114.160192 Jalloh, M. F., Li, W., Bunnell, R. E., Ethier, K. A., O'Leary, A., Hageman, K. M., ... Redd, J. T. (2018). Impact of Ebola experiences and risk perceptions on mental health in Sierra Leone, July 2015. BMJ Global Health, 3(2). https://doi.org/10.1136/bmjgh-2017-000471 Kojovic, Ben Hadid, Franchini, \& Schaer. (2019). Sensory Processing Issues and Their Association with Social Difficulties in Children with Autism Spectrum Disorders. Journal of Clinical Medicine, 8(10), 1508. https://doi.org/10.3390/jcm8101508 Kumazaki, H., Muramatsu, T., Kobayashi, K., Watanabe, T., Terada, K., Higashida, H., ... Kikuchi, M. (2020). Feasibility of autism-focused public speech training using a simple virtual audience for autism spectrum disorder. Psychiatry and Clinical Neurosciences, 74(2), 124-131. https://doi.org/10.1111/pcn.12949

Lai, M. C., Kassee, C., Besney, R., Bonato, S., Hull, L., Mandy, W., ... Ameis, S. H. (2019). 
Prevalence of co-occurring mental health diagnoses in the autism population: a systematic review and meta-analysis. The Lancet Psychiatry, 6(10), 819-829. https://doi.org/10.1016/S2215-0366(19)30289-5

Lever, A. G., \& Geurts, H. M. (2016). Psychiatric Co-occurring Symptoms and Disorders in Young, Middle-Aged, and Older Adults with Autism Spectrum Disorder. Journal of Autism and Developmental Disorders, 46(6), 1916-1930. https://doi.org/10.1007/s10803-016$2722-8$

Meltzer, A., \& Van De Water, J. (2017, January 1). The Role of the Immune System in Autism Spectrum Disorder. Neuropsychopharmacology, Vol. 42, pp. 284-298. https://doi.org/10.1038/npp.2016.158 Narzisi, A. (2020, April 1). Handle the autism spectrum condition during coronavirus (Covid-19) stay at home period: Ten tips for helping parents and caregivers of young children. Brain Sciences, Vol. 10. https://doi.org/10.3390/brainsci10040207 Neumeyer, A. M., Anixt, J., Chan, J., Perrin, J. M., Murray, D., Coury, D. L., ... Parker, R. A. (2019). Identifying Associations Among Co-Occurring Medical Conditions in Children With Autism Spectrum Disorders. Academic Pediatrics, 19(3), 300-306.

https://doi.org/10.1016/j.acap.2018.06.014

Peckett, H., Maccallum, F., \& Knibbs, J. (2016). Maternal experience of Lego Therapy in families with children with autism spectrum conditions: What is the impact on family relationships? Autism, 20(7), 879-887. https://doi.org/10.1177/1362361315621054 Roorda, D. L., Koomen, H. M. Y., Spilt, J. L., Thijs, J. T., \& Oort, F. J. (2013). Interpersonal behaviors and complementarity in interactions between teachers and kindergartners with a variety of externalizing and internalizing behaviors. Journal of School Psychology, 51(1), 143-158. https://doi.org/10.1016/j.jsp.2012.12.001 Rydzewska, E., Hughes-McCormack, L. A., Gillberg, C., Henderson, A., MacIntyre, C., Rintoul, J., \& Cooper, S. A. (2019). Prevalence of sensory impairments, physical and intellectual disabilities, and mental health in children and young people with self/proxyreported autism: Observational study of a whole country population. Autism, 23(5), 1201-1209. https://doi.org/10.1177/1362361318791279 